\title{
Systematic Review of Lay Responders Educational Outcomes to Identify Life-Threatening Bleeding
}

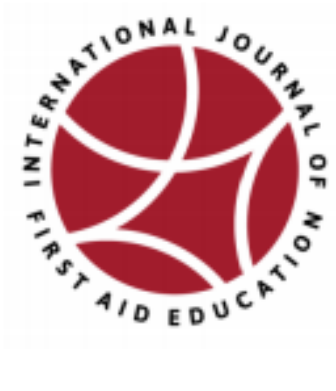

Jeffrey L. Pellegrino ${ }^{1}$, Stephen E. Smith ${ }^{1}$, Emma Banton ${ }^{1}$, Amita Sudhir ${ }^{2}$,

${ }^{1}$ University of Akron, ${ }^{2}$ University of Virginia Health System

Objective: Identify evidence of how lay responders can accurately identify life-threatening bleeding (LTB) from educational interventions.

Method: A systematic review process of the PubMed database to identify experimental and observational studies of educational interventions to identify LTB by lay responders. No exclusion regarding timeframe.

Abstracts needed to be in English.

Results: Three studies were identified, two with moderate risk of bias due to non-validation of measurement tool and one with a high risk of bias. All studies contributed to a notion that lay responders could identify a gross characteristic of LTB when presented visually.

Implications: A gap exists in evidence to suggest lay responders can accurately distinguish LTB from non-LTB in the field or with distractions (e.g., clothing, porous surfaces). A valid means of measuring this learning outcomes would allow for future research to be reviewed and for meta-analysis for educational purposes to distinguish quality within and between programs. Secondarily, it may serve as a proxy for survival behaviors in the field, as application of tourniquets is still rare. Educationally, multiple representations of LTB in a localized context can be used (online or face-to-face) to raise awareness of characteristics of LTB for future identification.

Keywords: Stop the Bleed, tourniquet, education, systematic review, early recognition

A severe hemorrhage in an out-of-hospital setting may lead to death faster than the expected time frame for medical professionals to arrive, let alone act (Goolsby et al., 2020; Lee et al., 2007; Passos et al., 2014). To counteract this, over one million laypersons and non-medical responders (e.g., police, school personnel) have now been trained to stop Life Threatening Bleeding (LTB) since the launch of the Stop the Bleed ${ }^{\circledR}(\mathrm{StB})$ campaign in the United States (American College of Surgeons, n.d.). Through these awareness and training experiences, specific knowledge and skills for tourniquet use and wound packing are taught and practiced (Goolsby et al., 2021). The Chain of Survival Behaviors (International Federation of Red Cross Red Crescent Societies, 2016) describes the domains of first aid education, in which "early recognition" is an essential link to initiating first aid - in this case, pressure on a bleeding wound (Goolsby, Jacobs, et al., 2018; Goolsby, Strauss-Riggs, et al., 2018).

The Stop the Bleed Educational Consortium (SBEC), a voluntary collaborative of clinicians, educators, and advocates from public, private, and non-governmental organizations, outlined three educational domains for the Stop the Bleed $\mathbb{R}$ educational program: first, to motivate learners to act when needed; second, to teach leaners to distinguish LTB from non-LTB at a first aid level; third, to teach learners how to apply pressure directly and use indirect pressure via a tourniquet, if amenable (Goolsby, Jacobs, et al., 2018). Accurate Pellegrino, et al. 2021 Creative Commons AttributionNonCommercial 4.0 International

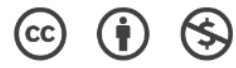


recognition of bleeding as non-LTB and LTB thus at a first aid level is a decision point for the prioritization of care.

SBEC recognized educational limitations of time with learners, the lack of learner background in medical competencies, the complexities of describing an LTB, and the stress of needing to recall any learning during an emergency, when they recommended simplicity in teaching learners to identify an LTB. They recommended discussion of the volume of blood loss (referencing an example of a $1 / 2$ can of soda, $6 \mathrm{oz} / \approx 175 \mathrm{ml}$ ) and flow of blood (continuous and steady). They also recommended against the use of other strategies: specifically, color of the blood, pulsatility, or specific injury patterns, because these may cause confusion in the learners. Their recommendations for LTB identification however were consensus based. Subsequently, during item development of the Stop the Bleed Educational Assessment Tool (SBEAT), it was noted that individuals shown pictures of various wounds and gifs of bleeding flow described them not only in terms of volume and flow, but also graphically (ex. big, puddle), and emotionally (ex. "[expletive] load, "oh [expletive]”) (Pellegrino et al., 2020).

As a reference, volume appears to be a popular understanding, but accurately assessing blood volume loss from a wound is not easily done by trained medical professionals nor non-medical professionals (Adkins et al., 2014; Beer et al., 2005; Dildy et al., 2004; Frank et al., 2010; Yoong et al., 2010). According to Phillips et al. (2020) and Tebruegge et al. (2009), laypersons overestimate small volumes and underestimate large volumes of blood. This phenomenon may then be problematic in education without evidence-based practices for estimation tools, techniques, or skill development in identifying LTB. Phillips et al. also identified a potential complicating factor in that the gender of the victim may influence estimation; in their study injuries in females were more likely to be underestimated than male victims and less likely to be classified as an LTB.

Sensitivity and specificity toward identification of LTB is important to initiating first aid. Obvious death from exsanguination may occur if sensitivity is low. There is less evidence to suggest that low specificity is dangerous (Duignan et al., 2018), because the person is still alive, suggesting that a lower threshold for action may be appropriate for lay responders to understand versus a finite set of factors.

Strauss-Riggs et al. (2020) and Goralnick et al. (2020) challenged training organizations and StB advocates to better assess these types of programs through process outcome measures, specifically learning objectives with standardized, valid, and reliable tools. Attention by organizations is needed as to whether training and assessment objectives match program goals, track use with validated assessment tools, and provide descriptive statistics on learner and instructor assessment tool scores in order to determine learner outcomes. We initiated a systematic review of the literature of first aid education to stop LTB to help training organizations identify and assess the evidence of educational (training) outcomes on the identification of LTB. The goal was to provide training organizations and educators evidencebased educational practices within the domain of early recognition of LTB. We looked at the question: Can people (laypersons) learning first aid, with any specific pedagogical approach, accurately identify (a.k.a. diagnose) an LTB in order to appropriately intervene with life-saving skills?

\section{Methods}

This review used the Preferred Reporting Items for Systematic Reviews and Meta-Analyses 2020 (PRISMA) to promote transparency of this review and future use by others (Page et al., 2021). We used the Population, Intervention, Control, Outcome (PICO) method to specify the goals of our systematic review. The participants included were laypersons learning to stop LTB, with the intervention being any pedagogical approach to identifying LTB. The comparison for this review is a control group or another pedagogical approach to identify LTB, and the outcome is accurate identification of LTB.

\section{Eligibility and inclusion criteria}


Published original research articles on the development of ability/skills of laypeople to identify LTB were included. Randomized control trials as well as observation trials were sought, with no exclusion toward the date published. Abstracts needed to be in English in order to be considered.

\section{Information sources}

Utilizing the PubMed database, because of its comprehensive cataloging and search features for healthcare including medical education, we created a search string using a combination of key words in titles and abstracts (tiab) and exploded Medical Subject Headings (MeSH; MAJR). During pilot inquiries, the "bleeding" MeSH term yielded too many irrelevant results to our research question. Once we added the MeSH term "education" we noted an increase in relevance. Similar searches of the ERIC electronic database did not retrieve any relevant results. Our final search strategy used:

(("life-threatening" OR fatal OR trauma*) AND (hemorrhage OR bleeding OR "blood loss"[tiab] OR estimat*[tiab] OR external [tiab]) AND (bystander OR "lay person" OR "general public" OR "lay rescuer")) OR (("Hemorrhage/diagnosis" [MAJR]) AND ("Blood Volume Determination/methods" [MAJR])) OR (("stop the bleed" AND education)).

This search was last run in PubMed on April 9, 2021.

\section{Study selection}

Using the Rayyan software for Systematic Reviews (Ouzzani et al., 2016), initial results (title and abstract) were independently reviewed by at least two blinded reviewers (Authors A, B, \& C) for inclusion. In order to be included, an article had to be marked "include" by at least two reviewers. A third reviewer would alleviate any conflicts in the decision, but this was not needed. Titles and abstracts with clearly unrelated content were "excluded" from further analysis $(n=313)$. Other reasons for exclusion were publication in a foreign language, wrong outcome, wrong study design, and wrong population, as noted in Figure 1.
Citations marked as "include" or "maybe" by either of the reviewers were included in the full text review to determine if they met all criteria for inclusion. After applying all inclusion and exclusion criteria, three articles were found to be relevant. (See Figure 1)

\section{Risk of Bias}

Two review authors (Authors C and D) independently assessed the risk of bias for the included study. We resolved disagreements by consensus or by consulting a third review author (Author A). For the observation study, we used the Risk of Bias Assessment Tool for Nonrandomized Studies (RoBANS) (Kim et al., 2013).

\section{Results}

Our search for layperson LTB identification yielded three results, see supplemental file. Goolsby et al. (2018) used an intervention of a 5minute video, which described LTB and application of a tourniquet $(\mathrm{n}=95)$ and compared it to a control group without any instruction ( $n=131$ ). For both groups, $90 \%$ of the participants correctly identified 4 or 5 of five extremity wound images drawn by a medical illustrator, correctly as LTB or non-LTB. This may provide some level of evidence that people intuitively can identify LTB in gross terms. The risk of bias was moderate due to the lack of a validated tool to discriminate LTB by learners.

Goolsby et al. (2021) used a didactic approach in three settings describing LTB. High school age participants $(n=248)$, after experiencing either an instructor led, online only, or a hybrid course were then asked to complete an assessment consisting of extremity wound images drawn by a medical illustrator. Nearly all the participants identified 4 


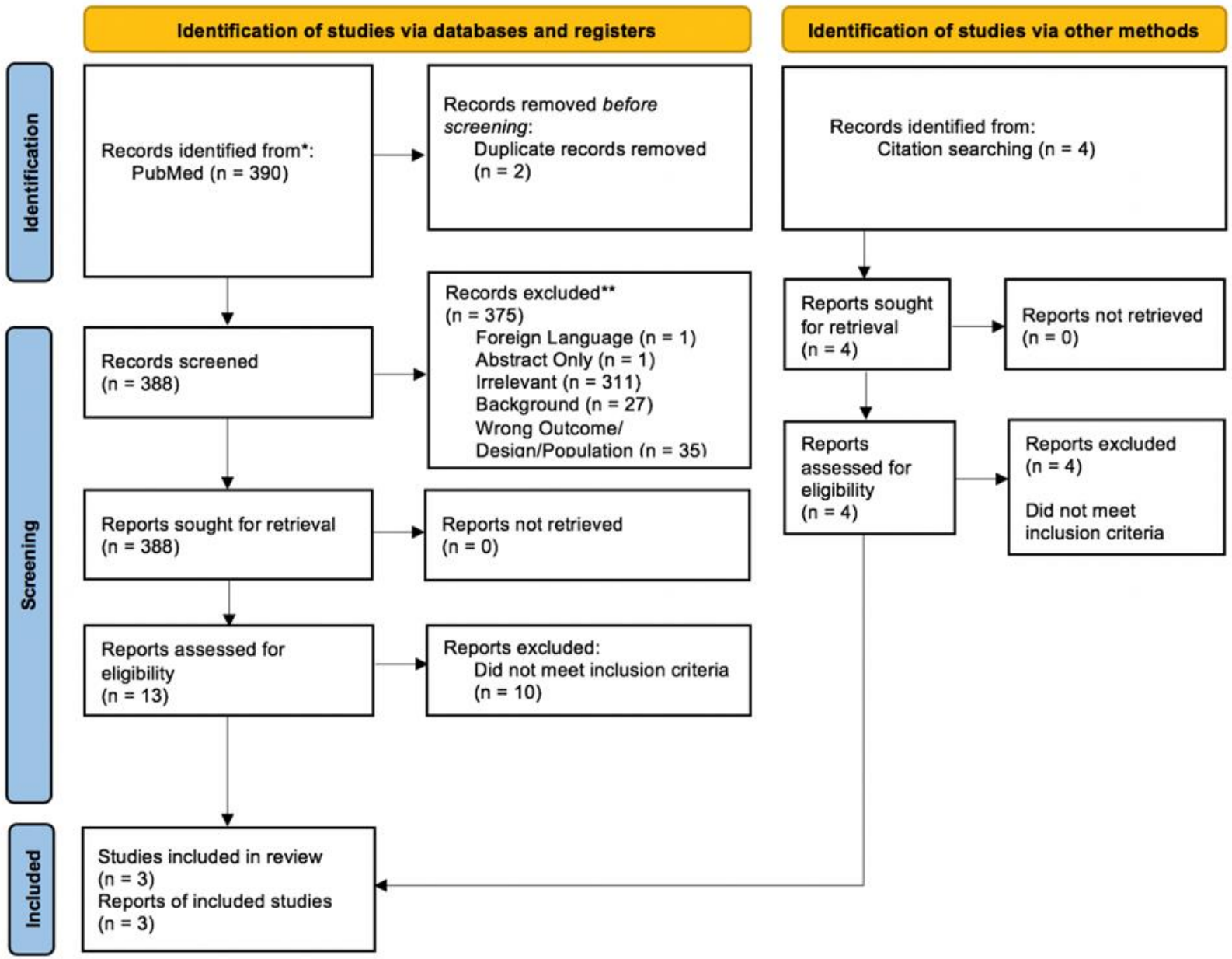

Figure 1: PRISMA Flow Diagram. Articles had no time limitation and last searched 9 April 21.

of the 5 images correctly $(99 \%$ in the instructor led and hybrid; $98 \%$ online only). No discussion about which item(s) were missed or why was mentioned. The risk of bias was moderate due to the lack of a validated tool to discriminate LTB by learners.

Gupta et al. (2019) started their education scenario with an LTB simulated with a high fidelity/lowcost synthetic perfused manikin appendage. They assessed that participants gained comfort and insight as to when bleeding was and was not controlled (aka LTB). Their use of a simulated pulsing blood flow from an open wound (also aided by the realistic nature of the manikin) was innovative when compared to an electronic simulator. This could also be construed as better than static devices (ex., moulage pool noodles or
PVC pipe) as their device allowed for bleeding control and performance assessment. Their Dynamic StB Model (DSM) used tubes and a hand pump operated manually by the educator. The DSM allowed for less expensive alternatives to electronically powered models, which could increase training locations, by using a hand pump and few parts. This provided a kinetic experience with visual cues sought by the authors to enhance verbal and pictorial descriptions/ evaluations of LTB. The Manikin was first beta-tested by $20 \mathrm{StB}$ instructors (five nurses and fifteen physicians). The main study was then conducted with 40 adults who were nonmedical, hospital administrative personnel, mostly females with a graduate degree in their late 30 's. They reported that $100 \%$ of the participants $(\mathrm{n}=40)$ felt the DSM "provided a better understanding of the rate of blood flow 
from an arterial wound than the commercial mannequin" (2019, p. 518).

The overall risk of bias for this study was determined to be high. The selection criteria of participants were not described. The presence of confounding variables was found to be high due to it being a pilot study with a very small sample size. The measurement of exposure was unclear as it is not clear if a standard measurement tool was used. The participants were not told they would be practicing on two models or assessing them, but blinding of the models was not possible, leading to unclear bias in the blinding of outcome assessments. All 40 participants returned assessments, leading to low bias in the outcome data, but no statistical analysis was performed, leading to a high bias in selective outcome reporting.

\section{Discussion}

Through this review, no direct evidence exists for lay responders to appropriately identify LTB or an educational intervention to instill the competency (knowledge, skills, or behaviors) in the field. This systematic inquiry into both the method of diagnosing LTB and teaching lay responders to identify LTB resulted in one study with a high risk of bias and two with moderate risk of bias. Although early recognition is essential to the Chain of Survival Behavior, most outcome-based educational studies involving laypersons identified in the review began with a scenario or description that was clearly an LTB, skipping early recognition and moving directly to the first aid domain.

The Gupta et al. results suggest the experience and outcomes may be extrapolated to lay responders initially identifying LTB using a physical model. The DSM manikin does not include features to represent bones and may require extensive cleaning to prevent mold/mildew/bacteria from growing within the tubes or the synthetic tissue. This may limit its usefulness in community education settings or educator willingness to maintain it appropriately. The system also depends on a hand pump and therefore would have varying levels of pulsatility, which must be taught to educators.

From an emotional standpoint, $90 \%$ of their participants reported feeling that the active bleeding from the DSM "heightened their urgency to perform StB techniques" (2019, p. 518). This emotional response based on a simulation corresponds to what Pellegrino et al. (2020) found during their focus group in describing LTB.

Estimating volume became a proxy for identification during our review process, although not exclusively, because it relates to the SBEC recommendation which is articulated in a finite amount (Goolsby, Jacobs, et al., 2018). It is important to note however that finite volume estimation of a bleeding wound is not the goal; the educational goal is to identify an action point for prioritizing the bleeding over other first aid actions. Essentially, the $6 \mathrm{oz}(\approx 175 \mathrm{ml})$ blood loss is a trigger point, so any education just needs to address this threshold. We believe there is some evidence initially identified in our systematic review to be extrapolated but excluded due to our a priori populations of lay responders. Studies of medical professionals might be extrapolated from the education to better frame the options and opportunities to develop a pedagogy to effectively and efficiently teach the identification of LTB.

The following studies identified through the literature search as methods of estimating blood loss, but not in lay responders are examples of potential cross over as they demonstrate the ability to quickly identify blood volumes through simple methods. Merlin et al. (2009) developed the MAR (Merlin-Alter-Raffel) method which uses fist size to estimate volume of blood on an impervious surface. Oriented palm-side down, one fist corresponded to $\sim 20 \mathrm{ml}$ of pooled blood based on the two authors' fist size. By quickly placing one fist next to another directly above the spill, the estimator can count the number of fists covering the area and multiply by 20 to gauge volume of pooled blood in milliliters. This method was taught to 74 medical professionals with varying 
levels of training and experience in under a minute. Small volumes $(75 \mathrm{ml})$ before instruction had an error of $120 \%$, and $73 \%$ for large volumes $(750 \mathrm{ml})$. Average error from the mean dropped by $76 \%$ for small volumes with $60 \%$ for the IQR, and $40 \%$ for large volumes with $45 \%$ for the IQR. This method is limited to a spill on an impervious surface and would therefore not be applicable to gauging on porous surfaces such as dirt, snow, or clothing. Significant increase in visual estimation was achieved through this quickly taught and implemented method, which was easily memorable regardless of medical experience. The MAR method is promising as it could be effectively and efficiently taught to laypersons; however, it still needs to be validated with laypersons, and limited in a first aid context because it doesn't address absorption volume. Additional validation of the role of fist size and description of technique is needed before this can be recommended. Modifications to this method, like the Palmer Method (Hettiaratchy \& Papini, 2004) for body surface estimation for burns, may exist which would then create a relationship between the amount of blood loss and the size of the person bleeding.

Another surface estimation technique that was taught to medical professionals used videos that were calibrated to different flow rates and wound sizes. SPOT GRADE, a bleeding severity scale, was validated to express six levels of bleeding (none, minimal, mild, moderate, severe, and extreme) in a population of surgeons $(n=14)$ (Spotnitz et al., 2018). The training consisted of 36 videos, which in the case of lay responders could be reduced if the goal to train was the threshold point of LTB. This method would also need validation in a lay responder population and is limited to surface estimation but is unique to include active flow of bleeding.

Using a 20-minute didactic session, Dildy et al. (2004) described mathematical formulas to calculate volume, illustrated volumes using common containers (ex. 12oz soda can), and simple rules of thumb in the saturation of common surgical materials. Clinical, medical, and nursing students and professionals $(n=53)$ went through a pre-test and post-test assessment, which showed a reduction in errors of estimation.

In a study of labor and delivery unit personnel $(\mathrm{n}=372)$, Toledo et al. (2010) experimentally (noncontrolled) examined pre/post blood loss estimation difference between live didactic and web-based didactic sessions. They used a series of stations with given amounts of blood in common containers and then the same amount on different materials (ex. laparotomy sponges and vaginal delivery drape). Participants were given a slide presentation that included "definition and risk factors for postpartum hemorrhage, the signs and symptoms of maternal hemorrhage, tools for estimating blood-loss, and the postpartum hemorrhage protocol" (2010, p. 400.e2). The didactic education provided resulted in significant improvements in postpartum hemorrhage and blood-loss estimations, with no significant differences between online and live session participants. No estimation of time on the didactics was provided, but the concept may be helpful for future layperson education on identifying LTB.

Overall, the evidence suggests that lay responders could identify a gross characteristic of LTB when presented visually. Recognition of LTB is a vital link however for first aid action. Phillips et al. (2020) observed the underestimation of blood loss by lay responders and noted an absence of direct evidence to suggest lay responders can identify LTB. This should challenge training organizations and course facilitators to apply some basic educational research to understand from a learner's perspective what is needed to recognize LTB, so as to take action.

\section{Implications}

In the absence of evidence-based guidelines or recommendations, we suggest the following pedagogical approaches be used and studied individually or in combination as to efficacy and 
efficiency in identifying LTB. Accepting SBEC's threshold recommendation of blood volume estimation of $6 \mathrm{oz}(\approx 175 \mathrm{ml})$ to initiate immediate action to stop the bleeding, volume needs to be contextualized for learners. This may be communicated with a pedagogy that includes pictures, classroom manipulatives/visual aids, and videos (Cumming \& Martinez-Taboada, 2020; Dildy et al., 2004; Merlin et al., 2009; Spotnitz et al., 2018; Toledo et al., 2010). These items should be authenticated to volume as well as contextual to situations in which learners might respond (ex. a bulky jacket or many layers of clothes). Pedagogically, exposure, context, comparison, and practice were all elements that corresponded with the visual aids (Cumming \& Martinez-Taboada, 2020; Dildy et al., 2004; Sukprasert et al., 2006).

Similarly, toward SBEC's recommendation for flow, which may be an initial sign of an LTB, videos or gifs can illustrate severity of bleeding to provide context to volume (Spotnitz et al., 2018). As seen in Gupta et al. (2019), flow and pulsatility can effectively be communicated through models. Having this reference may be amenable to situations/circumstances where volume estimation through calculation might be prohibitive (ex., violence, number of injured persons, etc.). Pedagogically, the feedback from models appears to have an impact on learners' outcomes, which we assume also had feedback from the education involved.

Other first aid signs \& symptoms of shock may also be applicable for internal LTB, as well as an indicator of severity in external bleeding (Frank et al., 2010). This also would expand the notion beyond external bleeding and help lay responders understand the severity of internal bleeding. The emotional elements of a lay responder feeling something is wrong, identified by Pellegrino, et al. (2020) as an "Oh Poop" moment, by being exposed to other signs and symptoms of shock and their eventual outcome, may help any underestimation of severity $6 \mathrm{oz}(\approx 175 \mathrm{ml})$ to be actionable.
Ethically, there is concern that creating psychological stress in educational settings to expose learners to realistic scenes via pictures, videos, models, and discussion may inadvertently cause distress. Educators need to be aware of their learners' vulnerabilities and developmentally approach identification of LTB. The use of stress inoculation pedagogy may be appropriate (Meichenbaum \& Novaco, 1985; Tsur et al., 2019).

Studies of stopping LTB by laypersons often ask about one's willingness to use a tourniquet, which in our opinion doesn't equal action, especially if there isn't evidence to suggest they can identify LTB outside of gross representations. Educators and training organizations need to be responsible for helping learners understand the reality of LTB situations, so they can have reasonable expectations or confidence of their reaction to a stressful situation. Although knowing what to do is a critical factor in the Chain of Survival Behaviors, it is predicated on recognizing the issue.

As technology continues to enhance emergency response, future identification of LTB may come from smartphones. Gerdessen et al. (2021) in a review of blood loss in surgery, noted the ability of a smartphone, via the camera and software, to estimate blood loss. This may not be the ideal solution but might be further developed along with intervention coaching. More likely, smartphones may be a conduit for which telecommunicators may be able to prompt bystanders without training to become immediate responders by having evidence-based questions to ask regarding bleeding (Barrus, 2018; Fraizer, 2019). From a technology perspective, NextGen 9-1-1 will allow telecommunicators to use and store pictures and videos from callers while providing emergency medical instructions. This again, requires additional research in this area of identifying LTB.

\section{Limitations}

Approaching this topic, we limited the scope to identification of LTB by laypersons. The SBEC 
group is the only group to offer a generalization of volume blood loss based on expert consensus $(6 \mathrm{oz} \approx 175 \mathrm{ml})$, as a threshold for lay responder immediate action. Accuracy of identification of LTB based on volume needs further description based on field evidence and/ or physiology (e.g., volume loss between an adult vs. an infant).

A lack of a validated means for which to assess a person's ability to recognize an LTB exists, limiting the current studies. The use of grossly depicted types of extremity bleeding didn't differentiate those with or without education, raising the question about the ability of the scale to differentiate or the intuitiveness of recognizing a lot of blood/ active bleeding. During the development of the SBEAT, the use of pictures and videos also didn't differentiate those with higher competencies.

\section{Conclusion}

This systematic review aimed to identify the need for standardized identification methods which could be easily taught to and then remembered by laypersons in actual emergencies/real-life scenarios. There is limited evidence that lay responders (trained and untrained) can identify characteristics or signs of LTB from a field context (e.g., stress, multiple injury, porous surfaces, clothes that mask wound). Most of the published research of courses that teach bleeding control techniques to laypeople begin with situations that are clearly life-threatening and assess what individuals do to stop the bleed. An evidence gap exists as to their ability to recognize

\section{References}

Adkins, A. R., Doohee Lee, D., \& Delilah, M. J. (2014). Accuracy of Blood Loss Estimations Among Anesthesia Providers. In AANA Journal (Vol. 82, Issue 4). www.aana.com/aanajournalonline

American College of Surgeons. (n.d.). Stop The Bleed. Retrieved June 6, 2021, from https://www.stopthebleed.org/

Barrus, B. (2018). Take A Stab - The Journal of Emergency Dispatch. The Journal of Emergency Dispatch. https://iaedjournal.org/take-a-stab/

Beer, H. L., Duvvi, S., Webb, C. J., \& Tandon, S. (2005). Blood loss estimation in epistaxis scenarios. Journal of Laryngology and Otology, 119(1), 16-18. https://doi.org/10.1258/0022215053222752

International Journal of First Aid Education, Vol. 4 Issue 2 
Cumming, S. H., \& Martinez-Taboada, F. (2020). Blood Loss Estimation in Small Animals and Assessment of a Pictorial Tool to Improve Accuracy in a Global Population of Veterinary Anesthesia Staff. Frontiers in Veterinary Science, 7, 212. https://doi.org/10.3389/fvets.2020.00212

Dildy, G. A., Paine, A. R., George, N. C., \& Velasco, C. (2004). Estimating blood loss: Can teaching significantly improve visual estimation? Obstetrics and Gynecology, 104(3), 601-606. https://doi.org/10.1097/01.AOG.0000137873.07820.34

Duignan, K. M., Lamb, L. C., DiFiori, M. M., Quinlavin, J., \& Feeney, J. M. (2018). Tourniquet use in the prehospital setting: Are they being used appropriately? American Journal of Disaster Medicine, 13(1), 37-43. https://doi.org/10.5055/ajdm.2018.0286

Fraizer, A. (2019). Mechanism Of Injury. The Journal of Emergency Dispatch. https://iaedjournal.org/mechanism-of-injury/

Frank, M., Schmucker, U., Stengel, D., Fischer, L., Lange, J., Grossjohann, R., Ekkernkamp, A., \& Matthes, G. (2010). Proper estimation of blood loss on scene of trauma: Tool or tale? Journal of Trauma Injury, Infection and Critical Care, 69(5), 1191-1195. https://doi.org/10.1097/TA.0b013e3181c452e7

Gerdessen, L., Meybohm, P., Choorapoikayil, S., Herrmann, E., Taeuber, I., Neef, V., Raimann, F. J., Zacharowski, K., \& Piekarski, F. (2021). Comparison of common perioperative blood loss estimation techniques: a systematic review and meta-analysis. In Journal of Clinical Monitoring and Computing (Vol. 35, Issue 2, pp. 245-258). Springer Science and Business Media B.V. https://doi.org/10.1007/s10877-020-00579-8

Goolsby, C. A., Rojas, L. E., Andersen, M., Charlton, N., Tilley, L., Pasley, J., Rasmussen, T. E., \& Levy, M. J. (2020). Potentially survivable fatal vascular access hemorrhage with tourniquet use: A postmortem analysis. Journal of the American College of Emergency Physicians Open, 1(6), 12241229. https://doi.org/10.1002/emp2.12201

Goolsby, C. A., Rojas, L. E., Rodzik, R. H., Gausche-Hill, M., Neal, M. D., \& Levy, M. J. (2021). High-School Students Can Stop the Bleed: A Randomized, Controlled Educational Trial. Academic Pediatrics, 21(2),

321-328. http://ezproxy.uakron.edu:2048/login?url=http://search.ebscohost.com/login.aspx?direct=true $\& \mathrm{db}=$ edo \&AN=148863008\&site=eds-live

Goolsby, C. A., Rouse, E., Rojas, L., Goralnick, E., Levy, M. J., Kirsch, T., Eastman, A. L., Kellermann, A., Strauss-Riggs, K., \& Hurst, N. (2018). Post-Mortem Evaluation of Potentially Survivable Hemorrhagic Death in a Civilian Population. Journal of the American College of Surgeons, 227(5), 502-506. http://10.0.3.248/j.jamcollsurg.2018.08.692

Goolsby, C. A., Strauss-Riggs, K., Klimczak, V., Gulley, K., Rojas, L., Godar, C., Raiciulescu, S., Kellermann, A. L., \& Kirsch, T. D. (2018). Brief, Web-based Education Improves Lay Rescuer Application of a Tourniquet to Control Life-threatening Bleeding. AEM Education and Training, 2(2), 154-161. https://doi.org/10.1002/aet2.10093 
Goolsby, C., Jacobs, L., Hunt, R. C., Goralnick, E., Singletary, E. M., Levy, M. J., Goodloe, J. M., Epstein, J. L., Strauss-Riggs, K., Seitz, S. R., Krohmer, J. R., Nemeth, I., Rowe, D. W., Bradley, R. N., Gestring, M. L., \& Kirsch, T. D. (2018). Stop the Bleed Education Consortium: Education program content and delivery recommendations. Journal of Trauma and Acute Care Surgery, 84(1), 205-210. https://doi.org/10.1097/TA.0000000000001732

Goralnick, E., Ezeibe, C., Chaudhary, M. A., McCarty, J., Herrera-Escobar, J. P., Andriotti, T., de Jager, E., Ospina-Delgado, D., Goolsby, C., Hunt, R., Weissman, J. S., Haider, A., Jacobs, L., Andrade, E., Brown, J., Bulger, E. M., Butler, F. K., Callaway, D., Caterson, E. J., \& Choudhry, N. K. (2020). Defining a Research Agenda for Layperson Prehospital Hemorrhage Control: A Consensus Statement. JAMA Network Open, 3(7), e209393-e209393. https://doi.org/10.1001/jamanetworkopen.2020.9393

Gupta, A., Villegas, C. v., Rosenberg, J., Winchell, R. J., Barie, P. S., \& Narayan, M. (2019). Advancing the Education of Stop the Bleed: Development of a Perfused Synthetic Cadaver Model. Journal of Surgical Research, 244, 516-520. https://doi.org/10.1016/j.jss.2019.06.041

Hettiaratchy, S., \& Papini, R. (2004). ABC of burns: Initial management of a major burn: II-assessment and resuscitation. BMJ : British Medical Journal, 329(7457), 101. https://doi.org/10.1136/BMJ.329.7457.101

International Federation of Red Cross Red Crescent Societies. (2016). International first aid and resuscitation guidelines 2016. International Federation of Red Cross Red Crescent Socieites. http://www.ifrc.org/Global/Publications/Health/First-Aid-2016-Guidelines_EN.pdf

Kim, S. Y., Park, J. E., Lee, Y. J., Seo, H.-J., Sheen, S.-S., Hahn, S., Jang, B.-H., \& Son, H.-J. (2013). Testing a tool for assessing the risk of bias for nonrandomized studies showed moderate reliability and promising validity. Journal of Clinical Epidemiology, 66, 408-414. https://doi.org/10.1016/j.jclinepi.2012.09.016

Lee, C., Porter, K. M., \& Hodgetts, T. J. (2007). Tourniquet use in the civilian prehospital setting. In Emergency Medicine Journal (Vol. 24, Issue 8, pp. 584-587). https://doi.org/10.1136/emj.2007.046359

Meichenbaum, D., \& Novaco, R. (1985). Stress inoculation: a preventative approach. Issues in Mental Health Nursing, 7(1-4), 419-435. http://search.ebscohost.com/login.aspx?direct=true\&db=cmedm\&AN=3854020\&site=ehost-live

Merlin, M. A., Alter, S. M., Raffel, B., \& Pryor, P. W. (2009). External blood loss estimation using the MAR Method. The American Journal of Emergency Medicine, 27(9), 1085-1090. https://doi.org/10.1016/j.ajem.2008.07.039

Ouzzani, M., Hammady, H., Fedorowicz, Z., \& Elmagarmid, A. (2016). Rayyan-a web and mobile app for systematic reviews. Systematic Reviews, 5(1). https://doi.org/10.1186/s13643-016-0384-4

Page, M. J., McKenzie, J. E., Bossuyt, P. M., Boutron, I., Hoffmann, T. C., Mulrow, C. D., Shamseer, L., Tetzlaff, J. M., Akl, E. A., Brennan, S. E., Chou, R., Glanville, J., Grimshaw, J. M., Hróbjartsson, A., Lalu, M. M., Li, T., Loder, E. W., Mayo-Wilson, E., McDonald, S., ... Moher, D. (2021). The PRISMA 
2020 statement: An updated guideline for reporting systematic reviews. International Journal of Surgery, 88. http://10.0.3.248/j.ijsu.2021.105906

Passos, E., Dingley, B., Smith, A., Engels, P. T., Ball, C. G., Faidi, S., Nathens, A., \& Tien, H. (2014). Tourniquet use for peripheral vascular injuries in the civilian setting. Injury, 45(3). https://doi.org/10.1016/j.injury.2013.11.031

Pellegrino, J. L., Charlton, N. P., \& Goolsby, C. (2020). "Stop the Bleed” Education Assessment Tool (SBEAT): Development and Validation. Cureus, 12(9), e10567. https://doi.org/10.7759/cureus.10567

Phillips, R., Friberg, M., Lantz Cronqvist, M., Jonson, C.-O., \& Prytz, E. (2020). Visual estimates of blood loss by medical laypeople: Effects of blood loss volume, victim gender, and perspective. PLOS ONE, 15(11), e0242096. https://doi.org/10.1371/journal.pone.0242096

Spotnitz, W. D., Zielske, D., Centis, V., Hoffman, R., Gillen, D. L., Wittmann, C., Guyot, V., Campos, D. M., Forest, P., Pearson, A., \& McAfee, P. C. (2018). The SPOT GRADE. Spine, 43(11), E664-E671. https://doi.org/10.1097/BRS.0000000000002447

Strauss-Riggs, K., Kirsch, T. D., Prytz, E., Hunt, R. C., Jonson, C., Krohmer, J., Nemeth, I., \& Goolsby, C. (2020). Recommended Process Outcome Measures for Stop the Bleed Education Programs. AEM Education and Training, aet2.10443. https://doi.org/10.1002/aet2.10443

Sukprasert, M., Choktanasiri, W., Ayudhya, N. I. N., Promsonthi, P., \& O-Prasertsawat, P. (2006). Increase accuracy of visual estimation of blood loss from education programme. Journal of the Medical Association of Thailand, 89(SUPPL. 4).

Tebruegge, M., Misra, I., Pantazidou, A., Padhye, A., Maity, S., Dwarakanathan, B., Donath, S., Curtis, N., \& Nerminathan, V. (2009). Estimating blood loss: Comparative study of the accuracy of parents and health care professionals. Pediatrics, 124(4). https://doi.org/10.1542/peds.2009-0592

Toledo, P., McCarthy, R. J., Burke, C. A., Goetz, K., Wong, C. A., \& Grobman, W. A. (2010). The effect of live and web-based education on the accuracy of blood-loss estimation in simulated obstetric scenarios. American Journal of Obstetrics and Gynecology, 202(4), 400.e1-400.e5. https://doi.org/10.1016/j.ajog.2009.10.881

Tsur, A. M., Binyamin, Y., Koren, L., Ohayon, S., Thompson, P., \& Glassberg, E. (2019). High Tourniquet Failure Rates Among Non-Medical Personnel Do Not Improve with Tourniquet Training, Including Combat Stress Inoculation: A Randomized Controlled Trial. Prehospital and Disaster Medicine, 34(3), 282-287. https://doi.org/10.1017/S1049023X19004266

Yoong, W., Karavolos, S., Damodaram, M., Madgwick, K., Milestone, N., Al-Habib, A., Fakokunde, A., \& Okolo, S. (2010). Observer accuracy and reproducibility of visual estimation of blood loss in obstetrics: How accurate and consistent are health-care professionals? Archives of Gynecology and Obstetrics, 281(2), 207-213. https://doi.org/10.1007/s00404-009-1099-8 [APA citation. Calibri (body), 11pt, 1.15 spaced, add space after paragraph] 